\title{
Studies on the Effects of Interphase Heat Exchange during Thermal Explosion in a Combustible Dusty Gas with General Arrhenius Reaction-Rate Laws
}

\author{
K. S. Adegbie and F. I. Alao \\ Department of Mathematical Sciences, Federal University of Technology, P.M.B. 704, Akure 340252, Nigeria
}

Correspondence should be addressed to K. S. Adegbie, kolasade33@yahoo.com

Received 21 September 2011; Accepted 5 December 2011

Academic Editor: Morteza Rafei

Copyright (C) 2012 K. S. Adegbie and F. I. Alao. This is an open access article distributed under the Creative Commons Attribution License, which permits unrestricted use, distribution, and reproduction in any medium, provided the original work is properly cited.

\begin{abstract}
A mathematical model for thermal explosion in a combustible dusty gas containing fuel droplets with general Arrhenius reaction-rate laws, convective and radiative heat losses, and interphase heat exchange between gas and inert solid particles is investigated. The objective of the study is to examine the effects of interphase heat exchange between the gas and solid particles on (i) ignition of reacting gas, (ii) accumulation of heat by the solid particles during combustion process (iii) evaporation of the liquid fuel droplets, and (iv) consumption of reacting gas concentration. The equations governing the physical model with realistic assumptions are stated and nondimensionalised leading to an intractable system of first-order coupled nonlinear differential equations, which is not amenable to exact methods of solution. Therefore, we present numerical solutions as well as different qualitative effects of varying interphase heat exchange parameter. Graphs and Table feature prominently to explain the results obtained.
\end{abstract}

\section{Introduction}

The concept of thermal explosion in combustible dusty gas containing fuel droplets is of great importance in safety aspect of nuclear facilities, furnaces, gas turbines and internal combustion engines, coal mine, and so forth. The essence of the inert solid particles in the combustible gas is to delay the ignition or explosion which could cause catastrophe. The phase of research on combustion and explosions that began at the end of the nineteenth century and continues to this day is associated with the invention of the internal combustion engine, with the development of explosive technology and of internal ballistics for artillery, and, in recent decade, with the extensive introduction of jet and diesel engines. In many 
respects, these have stimulated the rapid development of combustion science [1]. The procedure for thermal explosion in gases, which contain fuel droplets, has been of much interest. After Semenov [2] developed the basic theory of phenomenon of thermal explosion, models that are more complicated have been suggested in $[3,4]$.

The focus of this research work is therefore on the long-standing problem of thermal explosion and ignition in a combustible gas containing fuel droplets and its numerous applications to furnaces, gas turbines, and internal combustion engines [3-7]. Over recent years, the theoretical analysis of this problem has been performed mainly by the use of the geometrical asymptotic method of integral manifolds [8-17]. An alternative approach to the problem is to analyse the equations in some limiting cases. Klammer et al. [18] investigated ignition, combustion, and detonation processes in dusty gases with combustion reaction. The dusty gas was considered as a two-continuum medium taking into account transport effects in the phases and nonequilibrium chemical reactions. Two-dimensional problems of ignition and detonation were developed in a plane gallery caused by a supersonic inflow stream and heating of the closed end of the gallery are studied with the analytical method of catastrophe and two finite-difference numerical methods. Krainov and Shaurman [19] studied the limits of flame propagation in a gas with suspended inert particles in the presence of external heat removal. The mathematical model used was based on an unsteady heat-diffusion twotemperature model of gas combustion in the presence of inert particles. The problem was solved by a numerical method. A parametric analysis was performed, and critical values of the parameter that characterizes external heat removal were obtained. Dispersed-phase parameters were determined for which the two-temperature nature of the medium was insignificant. Thus, the critical conditions of flame propagation in the gas with inert particles were obtained. Comparison of the numerical and analytic solutions showed that they agreed satisfactorily with small particles of the dispersed phase. Ben-Dor and Igra [20] considered the relaxation zone behind normal shock waves in a reacting dusty gas. It was assumed that the gas is monatomic. The conservation equations for a suspension composed of an ionized gas and small solid dust particles were formulated and then solved numerically. The solution revealed that the presence of the dust has a significant effect on the postshock flow field. Because of the dust, the relaxation zone was longer than in the pure plasma case; the equilibrium values for the suspension pressure and density were higher than in the dust-free case, whereas the values obtained for the temperature, degree of ionization, and velocity was lower. The numerical solution was executed for shock Mach numbers ranging from 10 to 17. It was found that the thermal relaxation length for the plasma decreases rapidly with increasing shock Mach number, whereas the thermal relaxation length for the suspension increases slightly with increasing $M$. Gol'dshtein et al. [8] studied criteria for thermal explosion with reactant consumption in a dusty gas. The dynamical regimes of the system were classified as slow regimes, thermal explosion with delay, and thermal explosion (without delay). The critical transition conditions for the different dynamical regimes were analysed. They emphasized that the critical conditions for transition between slow regimes and explosion with delay was a thermal explosion limit. The Thermal explosion limit was described in the phase space by a so-called duck-trajectory. El-Sayed [21] investigated the critical conditions of the adiabatic explosion problem of a gas-solid (dusty gas) mixture. The definitions used for the homogeneous gas to determine criticality were used for the gas-solid mixture. The analysis revealed that the classical definition of the critical point can be adopted and modified to determine the critical condition in $\gamma-\eta$ and $\gamma-\theta$ domains. It was also found that using the definition of criticality as an inflection point in the critical trajectory in the $\theta-\eta$ plane gives the same results as given by the classical definition of criticality. It was interesting 
to see that the critical and maximum points in the gas temperature-concentration domain could coincide. It was found that the presence of a solid produces more than one critical temperature. The limiting cases of the problem were also offered. The numerical solution showed that the supercritical trajectory shows a thermal runaway for the gas over the solid at the end of reaction.

In this paper, an attempt has been made to extend the problem of thermal explosion in a combustible gas mixture with addition of inert solid particles and to generalise the problem based on temperature dependence of the reaction rate (i.e., Arrhenius Power-law model equation [22-24]) given as $K\left(T_{g}\right)=A_{0}\left(T_{g} / T_{g 0}\right)^{n} \exp \left(-E / R_{u} T_{g}\right)$ while taking into account convective and radiative heat losses, temperature dependence of density, and thermal conductivity of the gas. Therefore, a generalised physical model for thermal explosion in combustible dusty gas mixture containing fuel droplets is developed in the present paper. The main interest is focused on numerical solutions of the system of coupled nonlinear ordinary differential equations governing the physical model. Parametric analysis is performed, and the results show that the delay before ignition of the reacting gas, accumulation of heat by dusty particles, evaporation of liquid fuel droplets, and consumption of reacting gas concentration depend significantly on interphase heat exchange between the gas and solid particles.

\section{Mathematical Model}

In this study, we consider combustible dusty gas with addition of fuel droplets. Dusty gas is a combustible gas with addition of solid particles. It is assumed that the solid phase is inert, monosized, and uniformly heated, and the dusty gas is optically thick. The dependence of the convective heat transfer coefficient on both gas temperature and droplet radii is taken into account. Thermal conductivity of both monoatomic and polyatomic gases is proportional to $\sqrt{T_{g}}$. Therefore, we assume $\lambda_{g}\left(T_{g}\right)=\lambda_{g 0} \sqrt{T_{g} / T_{g 0}}$. The rate of reaction is based on generalised temperature-dependent Arrhenius's equation. Temperature dependence of the gas density and preexponential factor is given as $\rho_{g}\left(T_{g}\right)=\rho_{g 0} T_{g 0} / T_{g}$ and $A\left(T_{g}\right)=A_{0}\left(T_{g} / T_{g 0}\right)^{n}$, respectively. Following the realistic assumptions above, the system of governing equations has the form:

$$
\begin{aligned}
C_{p g} \phi_{g} \rho_{g 0} T_{g 0} T_{g}^{-1} \frac{d T_{g}}{d t}= & Q_{f} m_{f} \phi_{g} c_{f} A_{0} T_{g 0}^{-n} T_{g}^{n} \exp \left(\frac{-E}{R_{u} T_{g}}\right)-4 \pi R_{d} n_{d} \lambda_{g 0} \sqrt{\frac{T_{g}}{T_{g 0}}}\left(T_{g}-T_{g 0}\right) \\
& -4 \pi R_{d}^{2} n_{d} \sigma\left(T_{g}^{4}-T_{g 0}^{4}\right)-4 \pi R_{s} n_{s} \lambda_{s}\left(T_{g}-T_{s}\right), \\
\rho_{s} C_{p s} \frac{d T_{s}}{d t}= & 4 \pi R_{S} n_{s} \lambda_{s}\left(T_{g}-T_{s}\right), \\
\frac{d R_{d}}{d t}= & -\frac{1}{L \rho_{d}} \frac{\lambda_{g 0}}{R_{d}} \sqrt{\frac{T_{g}}{T_{g 0}}}\left(T_{g}-T_{g 0}\right)-\frac{1}{L \rho_{d}} \sigma\left(T_{g}^{4}-T_{g 0}^{4}\right), \\
\varphi_{g} \frac{d c_{f}}{d t}= & -\varphi_{g} c_{f} A_{0} T_{g 0}^{-n} T_{g}^{n} \exp \left(\frac{-E}{R_{u} T_{g}}\right)+\frac{4 \pi R_{d} n_{d} \lambda_{g 0}}{L m_{f}} \sqrt{\frac{T_{g}}{T_{g 0}}}\left(T_{g}-T_{g 0}\right) \\
& +\frac{4 \pi R_{d}^{2} n_{d} \sigma}{L m_{f}}\left(T_{g}^{4}-T_{g 0}^{4}\right) .
\end{aligned}
$$


The system (2.1)-(2.4) includes energy equations for the reacting gas (2.1) and solid particles (2.2), a mass equation for the liquid droplet (2.3) and a concentration equation for the reacting gas mixture (2.4). Initial conditions for the system:

$$
T_{g}(0)=T_{g 0}, \quad T_{s}(0)=T_{s 0}, \quad R_{d}(0)=R_{d 0}, \quad c_{f}(0)=c_{f 0} .
$$

\subsection{Nondimensional Analysis}

In this study, we introduce the following dimensionless variables:

$$
\theta_{g}=\frac{\left(T_{g}-T_{g 0}\right) E}{R_{u} T_{g 0}^{2}}, \quad \theta_{s}=\frac{\left(T_{g}-T_{s}\right) E}{R_{u} T_{s 0}^{2}}, \quad r=\frac{R_{d}}{R_{d 0}}, \quad \eta=\frac{c_{f}}{c_{f 0}}, \quad \tau=\frac{t}{t_{*}}
$$

We assume that at initial stage the temperature of gas and solid particles are the same, that is, $T_{g 0}=T_{s 0}$. Therefore, using (2.6) in (2.1)-(2.5), the dimensionless system of governing equations has the following form:

$$
\begin{aligned}
\left(1+\beta \theta_{g}\right)^{-1} \frac{d \theta_{g}}{d \tau}= & \delta \eta\left(1+\beta \theta_{g}\right)^{n} \exp \left(\frac{\theta_{g}}{1+\beta \theta_{g}}\right)-r\left\{\alpha_{1} \theta_{g} \sqrt{\left(1+\beta \theta_{g}\right)}+\alpha_{2} r\left[\left(1+\beta \theta_{d}\right)^{4}-1\right]\right\} \\
& -\alpha_{3}\left(\theta_{g}-\theta_{s}\right), \\
\frac{d \theta_{s}}{d \tau}= & \alpha_{3} \mu\left(\theta_{g}-\theta_{s}\right), \\
\frac{d r}{d \tau}= & -\frac{\mu^{*}}{r}\left\{\alpha_{1} \theta_{g} \sqrt{\left(1+\beta \theta_{g}\right)}+\alpha_{2} r\left[\left(1+\beta \theta_{g}\right)^{4}-1\right]\right\} \\
\frac{d \eta}{d \tau}= & -\alpha_{4} \delta \eta\left(1+\beta \theta_{g}\right)^{n} \exp \left(\frac{\theta_{g}}{1+\beta \theta_{g}}\right)+\psi r\left\{\alpha_{1} \theta_{g} \sqrt{\left(1+\beta \theta_{g}\right)}+\alpha_{2} r\left[\left(1+\beta \theta_{g}\right)^{4}-1\right]\right\}
\end{aligned}
$$

with the initial conditions:

$$
\theta_{g}(0)=0, \quad \theta_{s}(0)=0, \quad r(0)=1, \quad \eta(0)=1
$$


In (2.7)-(2.11), the following dimensionless parameters have been introduced:

$$
\begin{gathered}
\beta=\frac{R_{u} T_{g 0}}{E}, \quad B=\frac{L}{Q_{f}}, \quad \delta=\frac{E Q_{f} m_{f} C_{f 0} A_{0} t_{*}}{\rho_{g 0} C_{p g} R_{u} T_{g 0}^{2}} \exp \left(-\frac{E}{R_{u} T_{g 0}}\right), \quad \alpha_{1}=\frac{4 \pi R_{d 0} n_{d} \lambda_{g 0} t_{*}}{\rho_{g 0} C_{p g} \phi_{g}}, \\
\alpha_{2}=\frac{4 \pi R_{d 0}^{2} n_{d} \sigma T_{g 0}^{2} E t_{*}}{\rho_{g 0} C_{p g} \phi_{g} R_{u}}, \quad \alpha_{3}=\frac{4 \pi R_{s} n_{s} \lambda_{s} t_{*}}{\rho_{g 0} C_{p g} \phi_{g}}, \quad \alpha_{4}=B \psi, \quad \mu^{*}=\frac{\rho_{g 0} C_{p g} \phi_{g} R_{u} T_{g 0}^{2}}{4 \pi R_{d 0}^{3} n_{d} \rho_{d} E L} \\
\mu=\frac{\rho_{g 0} C_{p g} \phi_{g}}{\rho_{s} C_{p s}}, \quad \psi=\frac{\rho_{g 0} C_{p g} \phi_{g} R_{u} T_{g 0}}{E m_{f} C_{f 0} L} .
\end{gathered}
$$

Some special cases of the system of coupled nonlinear ordinary differential equations (2.7)(2.10) with initial conditions (2.11) and related problems have been studied for $n \neq 0$ and $\alpha_{3}=$ 0 (see, e.g., $[22,23,25]$ and the references therein). In the events that $n=0$ and parameters $\alpha_{1}, \alpha_{2}$, and $\alpha_{3}$ are varied, analyses have been performed both analytically and numerically in $([2,8-17,26,27]$, and references cited therein). In this study, attention is focused on the generalization of the problem and effect of the interphase heat exchange between the reacting gas and solid particles on the ignition of reacting gas, accumulation of heat by dusty particles, rate of evaporation of liquid fuel droplets, and depletion of reacting gas concentration.

\section{Numerical Computation}

In this section, we present numerical solutions of the system of governing equations (2.7)(2.10) with initial conditions (2.11). Meanwhile, before actual computation, we begin by deriving the exact integral for the system of ordinary differential equations as follows:

Multiplying (2.7) by $\alpha_{4}$, we get

$$
\begin{aligned}
\alpha_{4}\left(1+\beta \theta_{g}\right)^{-1} \frac{d \theta_{g}}{d \tau}= & \alpha_{4} \delta \eta\left(1+\beta \theta_{g}\right)^{n} \exp \left(\frac{\theta_{g}}{1+\beta \theta_{g}}\right) \\
& -\alpha_{4} r\left\{\alpha_{1} \theta_{g} \sqrt{\left(1+\beta \theta_{g}\right)}+\alpha_{2} r\left[\left(1+\beta \theta_{d}\right)^{4}-1\right]\right\}-\alpha_{3} \alpha_{4}\left(\theta_{g}-\theta_{s}\right)
\end{aligned}
$$

Adding (3.1) and (2.10) together gives

$$
\begin{aligned}
\alpha_{4}\left(1+\beta \theta_{g}\right)^{-1} \frac{d \theta_{g}}{d \tau}+\frac{d \eta}{d \tau}= & -\alpha_{4} r\left\{\alpha_{1} \theta_{g} \sqrt{\left(1+\beta \theta_{g}\right)}+\alpha_{2} r\left[\left(1+\beta \theta_{d}\right)^{4}-1\right]\right\} \\
& +\psi r\left\{\alpha_{1} \theta_{g} \sqrt{\left(1+\beta \theta_{g}\right)}+\alpha_{2} r\left[\left(1+\beta \theta_{d}\right)^{4}-1\right]\right\}-\alpha_{3} \alpha_{4}\left(\theta_{g}-\theta_{s}\right)
\end{aligned}
$$


Rearranging, we get

$$
\begin{aligned}
\alpha_{4}\left(1+\beta \theta_{g}\right)^{-1} \frac{d \theta_{g}}{d \tau}+\frac{d \eta}{d \tau}= & \left(\psi-\alpha_{4}\right) r\left\{\alpha_{1} \theta_{g} \sqrt{\left(1+\beta \theta_{g}\right)}+\alpha_{2} r\left[\left(1+\beta \theta_{d}\right)^{4}-1\right]\right\} \\
& -\alpha_{3} \alpha_{4}\left(\theta_{g}-\theta_{s}\right)
\end{aligned}
$$

From (2.8) and (2.9), we obtain

$$
\alpha_{3}\left(\theta_{g}-\theta_{s}\right)=\frac{1}{\mu} \frac{d \theta_{s}}{d \tau}\left\{\alpha_{1} \theta_{g} \sqrt{\left(1+\beta \theta_{g}\right)}+\alpha_{2} r\left[\left(1+\beta \theta_{g}\right)^{4}-1\right]\right\}=-\frac{r}{\mu^{*}} \frac{d r}{d \tau} .
$$

Substituting (3.4) into (3.3) yields

$$
\alpha_{4}\left(1+\beta \theta_{g}\right)^{-1} \frac{d \theta_{g}}{d \tau}+\frac{d \eta}{d \tau}=\left(\frac{\alpha_{4}-\psi}{\mu^{*}}\right) r^{2} \frac{d r}{d \tau}-\frac{\alpha_{4}}{\mu} \frac{d \theta_{s}}{d \tau}
$$

Integrating (3.5) with respect to $\tau$, we obtain

$$
\frac{\alpha_{4}}{\beta} \ln \left(1+\beta \theta_{g}\right)+\eta=\left(\frac{\alpha_{4}-\psi}{3 \mu^{*}}\right) r^{3}-\frac{\alpha_{4}}{\mu} \theta_{s}+N,
$$

where $N$ is constant of integration.

Applying the initial condition (2.11) in (3.6), we get

$$
N=1-\frac{\psi}{3 \mu^{*}}(B-1), \quad \text { since } \alpha_{4}=B \psi
$$

Using (3.7) in (3.6) gives exact integral of the system of equations (2.7)-(2.10) as

$$
\eta=\eta\left(\theta_{g}, \theta_{s}, r\right)=1-\psi\left\{\frac{B}{\beta} \ln \left(1+\beta \theta_{g}\right)-\left(\frac{1-B}{3 \mu^{*}}\right)\left(1-r^{3}\right)+\frac{B}{\mu} \theta_{s}\right\}
$$

Now, by substituting $\eta$ in (3.8) into (2.7), it is possible to obtain the following equation:

$$
\begin{aligned}
\frac{d \theta_{g}}{d \tau}= & \delta\left\{1-\psi\left[\frac{B}{\beta} \ln \left(1+\beta \theta_{g}\right)-\left(\frac{1-B}{3 \mu^{*}}\right)\left(1-r^{3}\right)+\frac{B}{\mu} \theta_{s}\right]\right\}\left(1+\beta \theta_{g}\right)^{n+1} \exp \left(\frac{\theta_{g}}{1+\beta \theta_{g}}\right) \\
& -r\left(1+\beta \theta_{g}\right)\left\{\alpha_{1} \theta_{g} \sqrt{\left(1+\beta \theta_{g}\right)}+\alpha_{2} r\left[\left(1+\beta \theta_{d}\right)^{4}-1\right]\right\}-\alpha_{3}\left(1+\beta \theta_{g}\right)\left(\theta_{g}-\theta_{s}\right) .
\end{aligned}
$$

The resulting systems of (2.8), (2.9), and (3.9) are coupled nonlinear differential equations and do not possess closed form solutions. Therefore, a numerical technique based on finite difference approximation is used to solve the problems. The effect of the interphase heat 
exchange parameter is adequately examined on the ignition time, combustible gas temperature, solid particle temperature, droplet radius, and combustible gas concentration as shown in the figures.

\section{Results}

The general characteristic features of Figures 1-4 show time histories of dimensionless gas temperature $\theta_{g}$, solid particle temperature $\theta_{s}$, gas concentration $\eta$ and liquid-fuel droplet radius $r$ for typical practical rate of reactions with various values of heat exchange parameter $\alpha_{3}$ when $\delta=0.3, \beta=0.05, B=0.008, \mu^{*}=5, \psi=0.3, \alpha_{1}=3 E-04, \alpha_{2}=2 E-05, \mu=1$. The qualitative impacts of interphase heat exchange between gas and solid particles $\alpha_{3}$ in this study are ascertained in the figures.

We notice in Figure 1 the occurrence of convectional thermal explosions in sensitized, Arrhenius, and bimolecular rate of reactions, respectively. Meanwhile in bimolecular rate of reaction, when $\alpha_{3}=1.0$, it is quite interesting to note that the problem has a quasi-steady solution after explosion. In this case, the medium is no longer homogeneous and diffusion term has to be taken into consideration. The medium could not be regarded as well stirred. Of a particular interest is the notable delay in the ignition time as the interphase heat exchange between gas and solid particles increases.

In Figure 2, it is observed that the heat exchange between the gas and solid particles raises the temperature of the solid particles at different time for sensitized, Arrhenius, and bimolecular rate of reactions. This implies that the solid particles accumulate heat over a period of time from reacting gas, since the solid particles are inert.

Figure 3 shows that the size of the liquid-fuel droplet radius significantly reduces as heat exchange between the gas and solid particles increases for sensitized, Arrhenius, and bimolecular rate of reactions. This reveals that the rate of evaporation of the liquid-fuel droplet substantially depends on heat exchange between the reacting gas and solid particles. We note that when $\alpha_{3}=0.5$ and $\alpha_{3}=1.0$, the liquid-fuel droplet completely evaporated just before ignition.

Figure 4 reveals a remarkable reduction in gas concentration as heat exchange between the gas and solid particles increases for sensitized, Arrhenius, and bimolecular rate of reactions. This depicts depletion of gas concentration during the combustion process.

In Figure 5, we present variation of $\theta_{g}, \theta_{s}, r$, and $\eta$ with numeric exponent for various values of $\alpha_{3}$ at ignition point. Important facts to note from these results are the following.

(i) As can be seen from Figures 5(a), 5(c), and 5(d), an increase in interphase heat exchange between the gas and solid particles causes significant reduction in the values of gas temperature, droplet radius, and gas concentration whereas in Figure $5(\mathrm{~b})$ it raises the value of solid particle temperature at ignition point for sensitized, Arrhenius, and bimolecular reaction rates, respectively.

(ii) It is also obvious from Figures 5(a) and 5(b) that an increase in numeric exponent $n$ ascertains reduction in the values of gas and solid particles temperature at ignition point, whereas in Figures 5(d) and 5(c) we observe increment in the values of gas concentration and insignificant reduction in the values of droplet radius at ignition point, respectively.

Table 1 shows the variation of dimensionless ignition times $\tau_{i g}$ with interphase heat exchange parameter $\alpha_{3}$ for typical sensitized, Arrhenius, and bimolecular rate of reactions at 


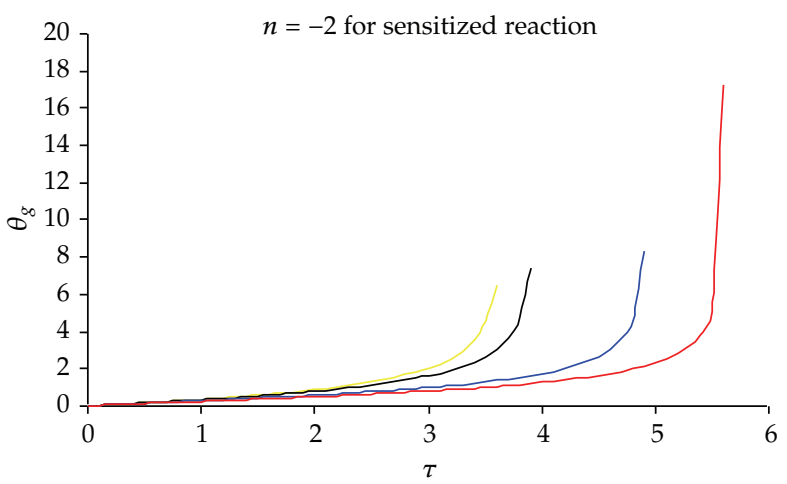

(a)

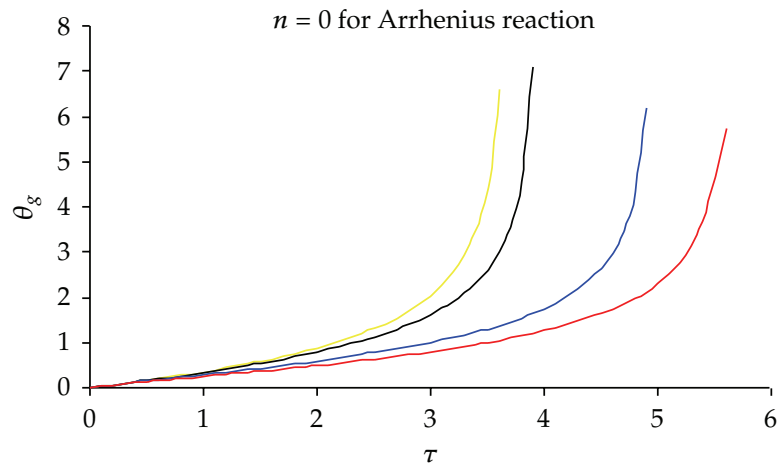

(b)

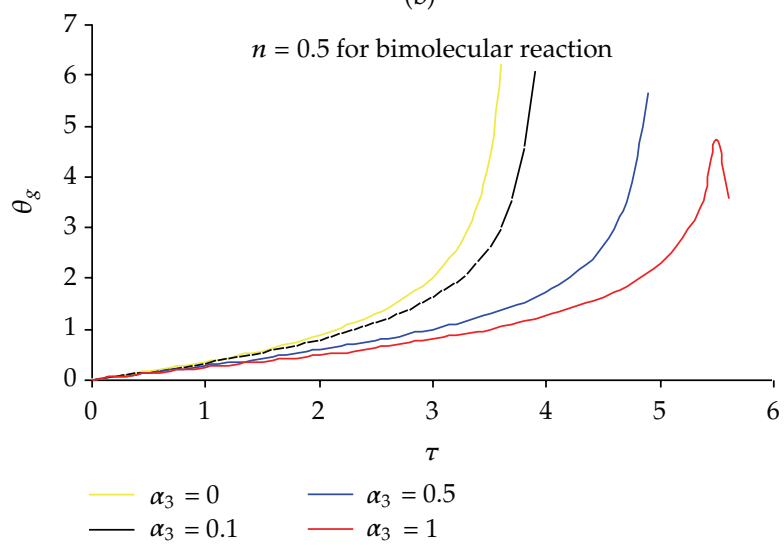

(c)

Figure 1: Variation of dimensionless combustible gas temperature with interphase heat exchange parameters when $n=\{-2,0,0.5\}$.

ignition point. It is significant to note from the table that increase in heat exchange between the gas and solid particles causes a notable delay in the ignition time of the combustible gas mixture for sensitized, Arrhenius, and bimolecular rate of reactions. The results also reveal that responsiveness to delay in ignition time is characterized by numeric exponent, which means that delay is more enunciate in sensitized reaction compared to Arrhenius and bimolecular reactions, respectively. 


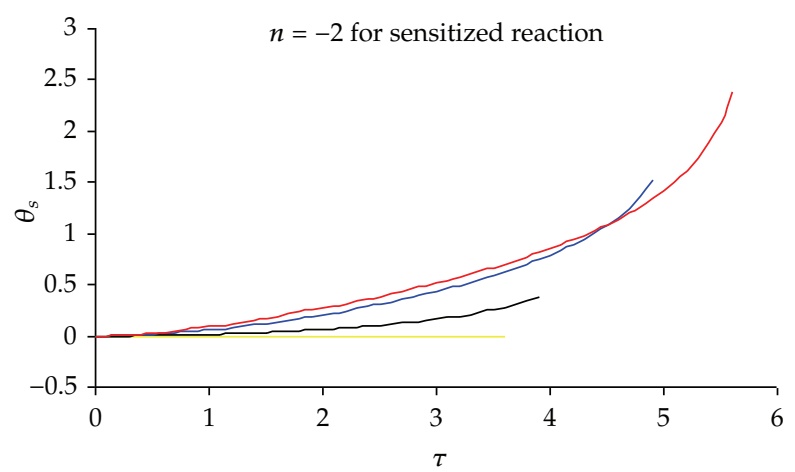

(a)

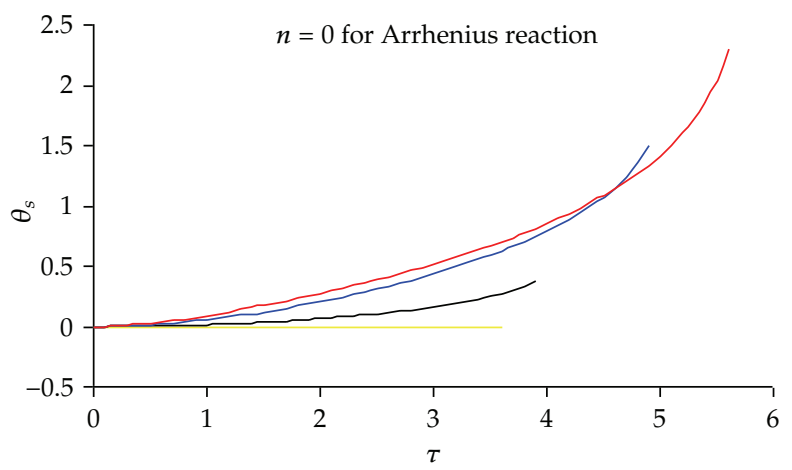

(b)

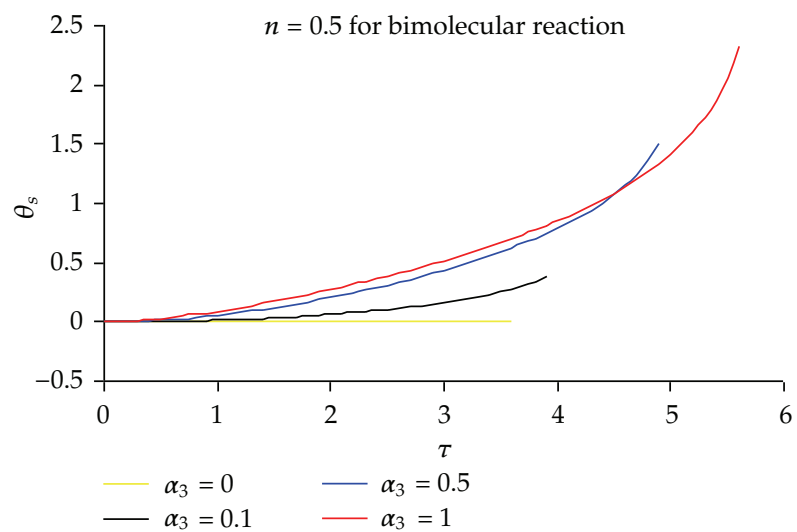

(c)

Figure 2: Variation of dimensionless solid particle temperature with interphase heat exchange parameters when $n=\{-2,0,0.5\}$.

\section{Conclusion}

The problem of thermal explosion in combustible dusty gas mixtures containing fuel droplets have been extended to permit a more general temperature dependent rate of reaction for most typical practical reactions under physically reasonable assumptions. The mathematical formulation involves a system of four highly nonlinear ordinary differential equations, 


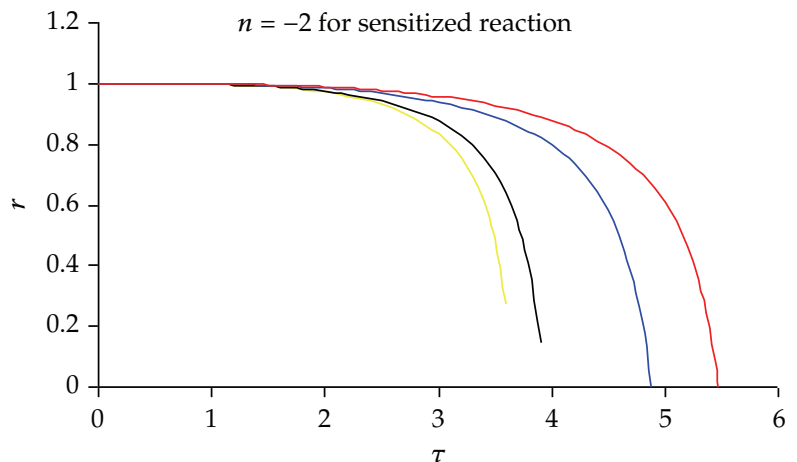

(a)

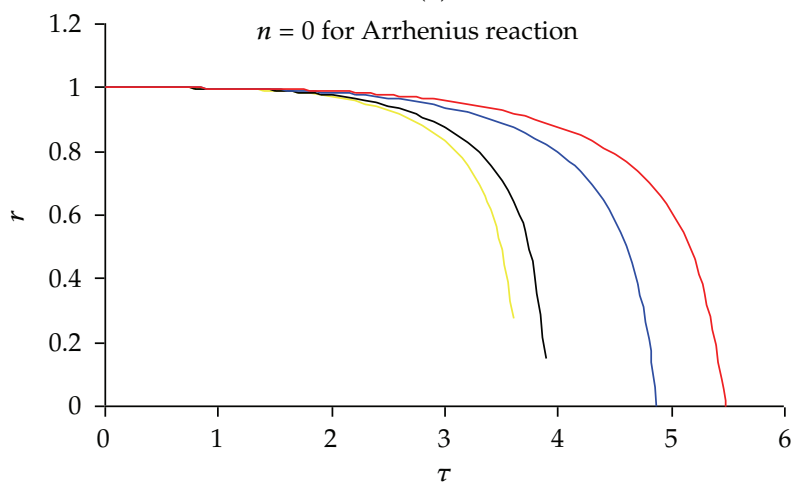

(b)

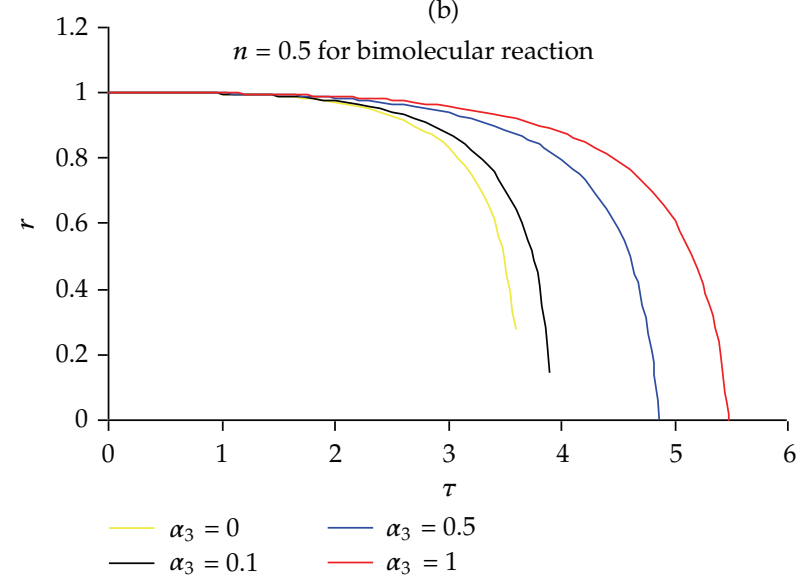

(c)

Figure 3: Variation of dimensionless droplet radius with interphase heat exchange parameters when $n=$ $\{-2,0,0.5\}$.

which was solved numerically by finite difference approximation scheme. In multiple phase processes, interphase heat exchange plays the role of heat losses in homogeneous combustible gas mixtures. The model with heat losses provides more detailed information about the effects of interphase heat exchange and numeric exponent characterising sensitized, Arrhenius, and bimolecular rate of reactions. The study revealed that interphase heat exchange 


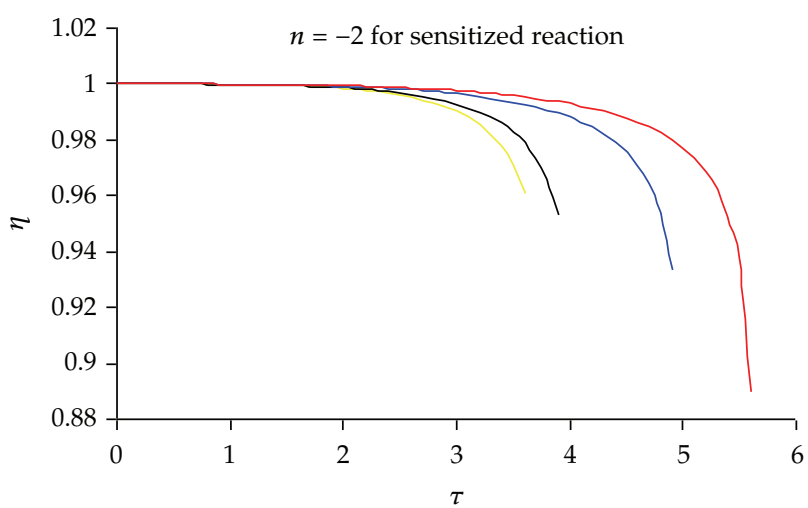

(a)

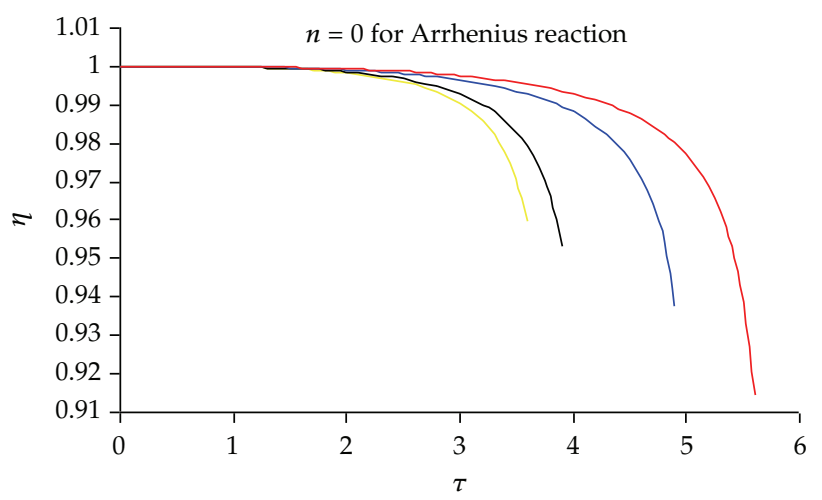

(b)

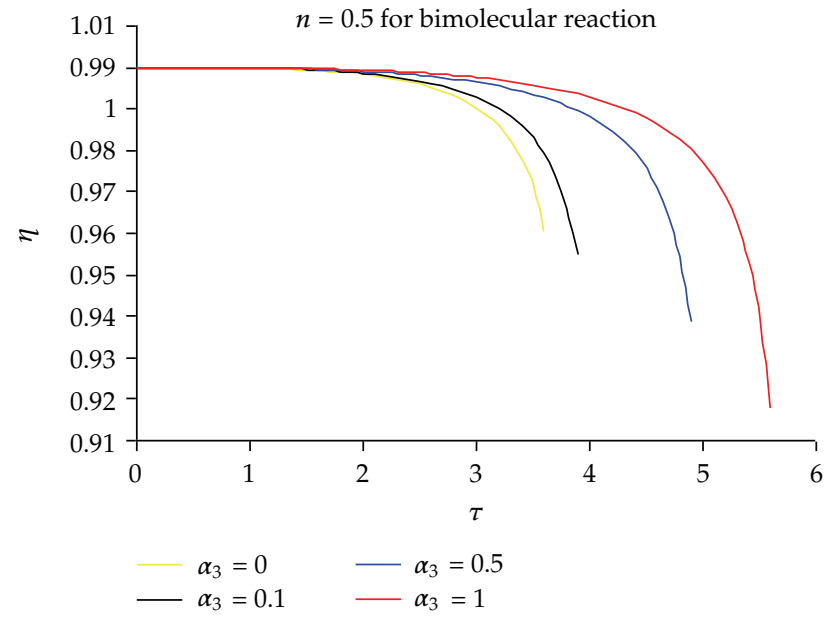

(c)

Figure 4: Variation of dimensionless combustible gas concentration with interphase heat exchange parameters when $n=\{-2,0,0.5\}$. 


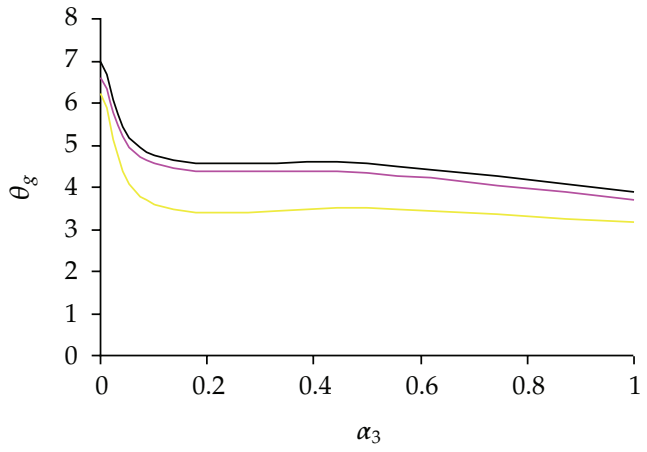

(a)

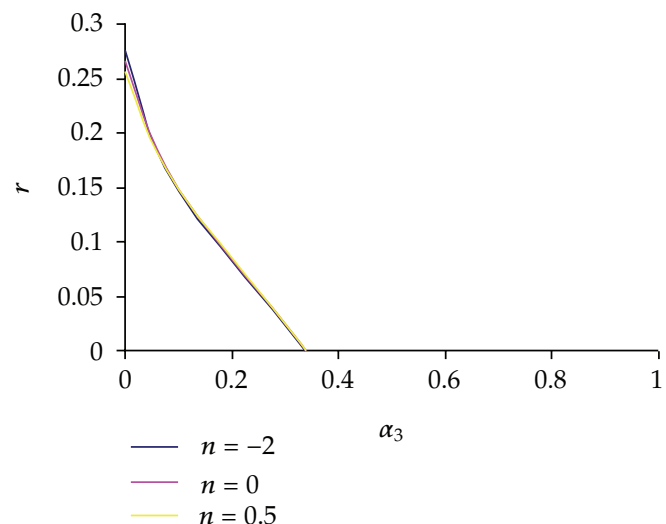

(c)

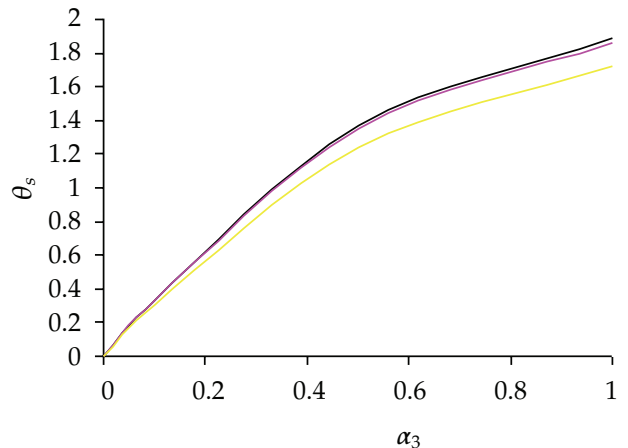

(b)

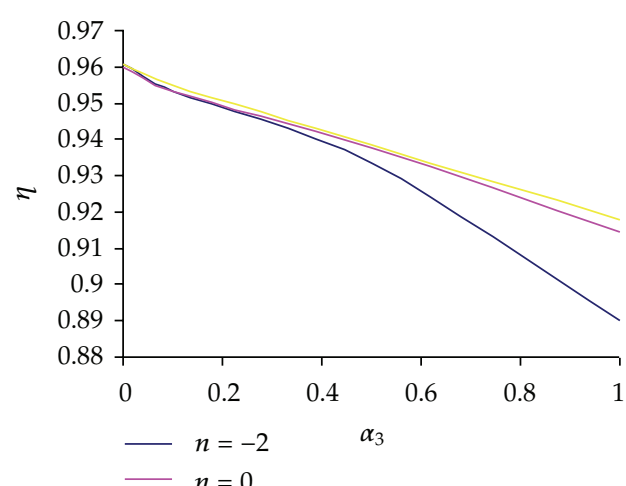

(d)

Figure 5: Variation of $\theta_{g}, \theta_{s}, r$, and $\eta$ with numeric exponent for various values of $\alpha_{3}$ at ignition point.

Table 1: Variation of ignition times with interphase heat exchange parameter at ignition point when $n=$ $\{-2,0,0.5\}$.

\begin{tabular}{lcccc}
\hline$n$ & & & & \\
$\tau_{i g}$ & & \\
& $\alpha_{3}=0$ & $\alpha_{3}=0.1$ & $\alpha_{3}=0.5$ & $\alpha_{3}=1.0$ \\
\hline-2.0 & 4.567872 & 4.938240 & 5.296256 & 7.160448 \\
0.0 & 4.074048 & 4.444416 & 5.555520 & 6.296256 \\
0.5 & 3.950592 & 4.320960 & 5.432064 & 6.049344 \\
\hline
\end{tabular}

between gas and solid particles, and numeric exponent play important roles in determining the following:

(1) occurrence of convectional thermal explosions and quasi-steady solution after explosion.

(2) significant delay in ignition time.

(3) reduction in inert solid particles temperature.

(4) remarkable reduction in the size of the liquid-fuel droplet leading to complete evaporation.

(5) Depletion of gas concentration during the combustion process. 
The results highlighted above do guarantee safety in practical explosion and combustion devices.

\section{Nomenclature}

English Symbols

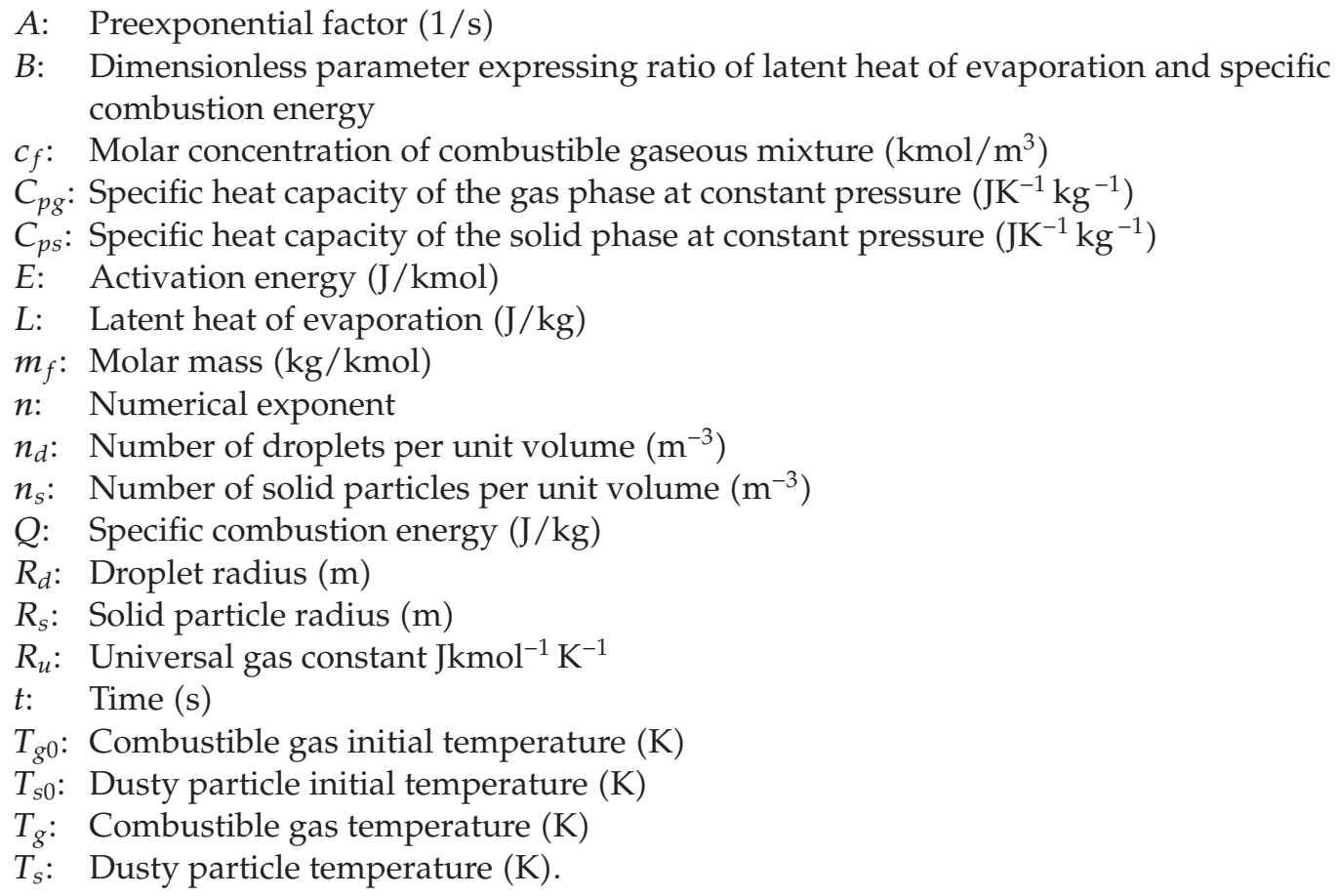

\section{Greek Symbols}

$\rho$ : Density of the combustible gaseous mixture $\left(\mathrm{kg} / \mathrm{m}^{3}\right)$

$\lambda$ : Thermal conductivity $\left(\mathrm{Wm}^{-1} \mathrm{~K}^{-1}\right)$

$\varphi$ : Volumetric phase content (dimensionless)

$\sigma$ : Stefan-Boltzmann's constant

$\beta$ : Dimensionless activation energy

$\tau$ : Dimensionless time

$\delta$ : Dimensionless parameter expressing reciprocal of the characteristic time for adiabatic temperature rise

$\alpha_{1}$ : Dimensionless parameter expressing heat loss via convection from gas phase

$\alpha_{2}$ : Dimensionless parameter expressing heat loss via radiation from gas phase

$\alpha_{3}$ : Dimensionless parameter expressing interphase heat exchange between gas and solid particle

$\mu$ : Dimensionless parameter expressing energy needed to transfer heat from gas phase to solid phase

$\mu^{*}$ : Dimensionless parameter expressing energy needed to evaporate all fuel droplets

$\psi$ : Dimensionless parameter expressing energy needed to consume all gas concentration. 


\section{Subscripts}

0: Initial

g: Gas mixture

$f$ : Combustible gas component of the mixture (fuel)

$d$ : Liquid droplets

$p$ : Constant pressure

ig: Ignition.

\section{References}

[1] Ya. B. Zeldovich, G. I. Barenblatt, V. B. Librovich, and G. M. Makhviladze, The Mathematical Theory of Combustion and Explosions, Consultants Bureau, New York, NY, USA, 1985.

[2] N. N. Semenov, Some Problems of Chemical Kinetics and Reactivity, vol. 1\&2, Pergamon Press, New York, NY, USA, 1958.

[3] K. K. Kuo, Principle of Combustion, John Wiley \& Sons, New York, NY, USA, 1986.

[4] J. J. Gray, Linear Differential Equations and Group Theory from Riemann to Poincaré, Birkhäuser, Boston, Mass, USA, Second edition, 2000.

[5] J. B. Heywood, Internal Combustion Engine Fundamentals, McGraw-Hill, Singapore, International edition, 1988.

[6] R. Stone, Introduction to Internal Combustion Engines, Macmillan, New York, NY, USA, 1992.

[7] J. Warnatz, U. Mass, and R. W. Dibble, Combustion: Physical and Chemical Fundamentals, Modelling and Stimulation, Experiments, Pollutants Formation, Springer, Berlin, Germany, 3rd edition, 2001.

[8] V. Gol'dshtein, A. Zlnoviev, V. Sobolev, and E. Shchepakina, "Criterion for thermal explosion with reactant consumption in a dusty gas," Proceeding of Royal Society of London A, vol. 452, no. 1952, pp. 2103-2119, 1996.

[9] I. Goldfarb, S. Sazhin, and A. Zinoviev, "Delayed thermal explosion in flammable gas containing fuel droplets: asymptotic analysis," Journal of Engineering Mathematics, vol. 50, no. 4, pp. 399-414, 2004.

[10] I. Goldfarb, V. Gol'dshtein, and A. Zinoviev, "Delayed thermal explosion in porous media: method of invariant manifolds," IMA Journal of Applied Mathematics, vol. 67, no. 3, pp. 263-280, 2002.

[11] I. Goldfarb and A. Zinoviev, "A study of delayed spontaneous insulation fires," Physics Letters, Section A, vol. 311, no. 6, pp. 491-500, 2003.

[12] I. Goldfarb, V. Gol'dshtein, J. B. Greenberg, and G. Kuzmenko, "Thermal explosion in a droplet-gas cloud," Combustion Theory and Modelling, vol. 4, no. 3, pp. 289-316, 2000.

[13] I. Goldfard, V. Gol'dshtein, G. Kuzmenko, and S .S. Sazhin, "Thermal radiation effect on thermal explosion in a gas containing fuel droplets," Combustion Theory and Modelling, vol. 3, pp. 769-787, 1999.

[14] V. Gol'dshtein and V. Sobolev, "Integral manifolds in chemical kinetics and combustion," in Singularity Theory and Some Problems of Functional Analysis, pp. 73-92, American Mathematical Society, Providence, RI, USA, 1992.

[15] V. Gol'dshtein, I. Goldfard, I. Shreiber, and A. Zinoviev, "Oscillations in a combustible gas bubble," Combustion Theory and Modelling, vol. 2, pp. 1-17, 1998.

[16] I. Goldfarb, V. Gol'dshtein, D. Katz, and S. Sazhin, "Radiation effect on thermal explosion in gas containing evaporated fuel droplets," International Journal of Thermal Sciences, vol. 46, pp. 358-370, 2007.

[17] V. Gol'dshtein and A. Zinoviev, "Thermal explosion in multiple phase media," Nonlinear Analysis, vol. 30, no. 8, pp. 4771-4780, 1997.

[18] J. Klammer, R. Klemens, V. P. Korobeinikov, V. V. Markov, I. S. Men'shov, and P. Wolanski, “On ignition and unsteady flows of dusty gases with combustion reactions," Combustion Science and Technology, vol. 142, no. 1, pp. 81-90, 1999.

[19] A. Yu. Krainov and V. A. Shaurman, "On the limits of flame propagation in a dusty gas," Combustion, Explosion and Shock Waves, vol. 33, no. 4, pp. 403-408, 1997.

[20] G. Ben-Dor and O. Igra, "The relaxation zone behind normal shock waves in a reacting dusty gas. I Monatomic gases," Journal of Plasma Physics, vol. 27, no. 3, pp. 377-395, 1982.

[21] S. A. El-Sayed, "Adiabatic thermal explosion of a gas-solid mixture," Combustion Science and Technology, vol. 176, no. 2, pp. 237-256, 2004. 
[22] K. S. Adegbie, A mathematical model of thermal explosion in a combustible gas containing fuel droplets, Ph.D. thesis, Lautech, Ogbomoso, Nigeria, 2008.

[23] K. S. Adegbie, "On delay-type behaviour of thermal explosion in a combustible gas mixture containing fuel droplets with arrhenius power-law model," Journal of the Nigerian Association of Mathematical Physics, vol. 68, pp. 69-82, 2008.

[24] International Union of Pure and Applied Chemistry (IUPAC) Goldbook Definition of Modified Arrhenius Equation, http:/ /en.wikipedia.org/wiki/Arrhenius/equation.

[25] K. S. Adegbie, "Some remarks on Arrhenius Power-law Model for thermal explosion in a combustible gas mixture containing fuel droplets," Ife Journal of Science, vol. 10, no. 1, pp. 358-370, 2008.

[26] R. O. Ayeni, O. Otolorin, O. T. Ayodele, and K. S. Adegbie, "Effect of frank-kamenetskii parameter on thermal explosion of flammable gas containing fuel droplets," Ife Journal Of Science, vol. 9, no. 1, pp. 55-62, 2005.

[27] D. A. Frank-Kamenetskii, Diffusion and Heat Transfer in Chemical Kinetics-Theory of Thermal Explosions, Plenum Press, New York, NY, USA, 1969. 


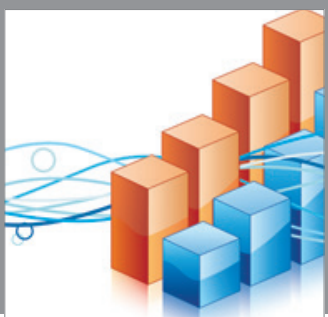

Advances in

Operations Research

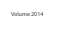

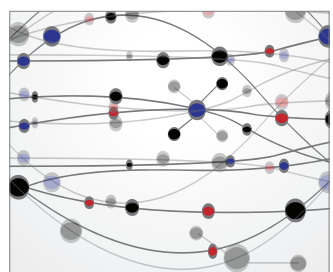

\section{The Scientific} World Journal
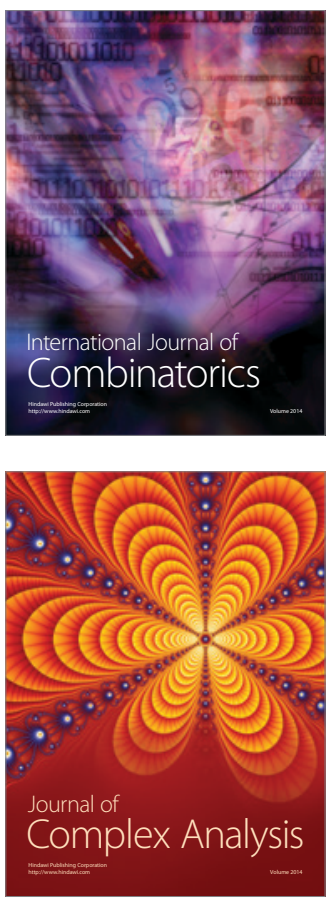

International Journal of

Mathematics and

Mathematical

Sciences
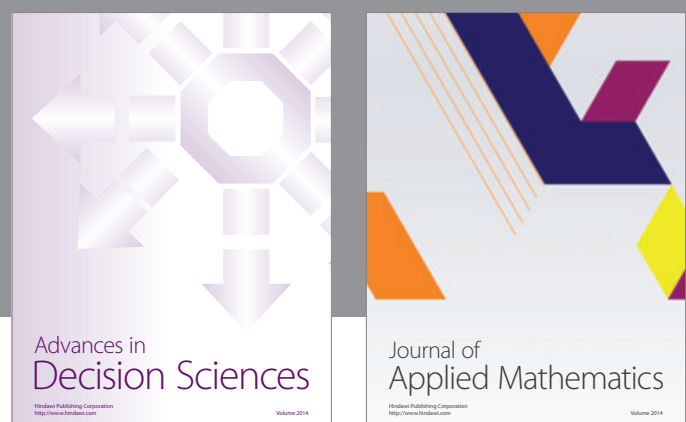

Journal of

Applied Mathematics
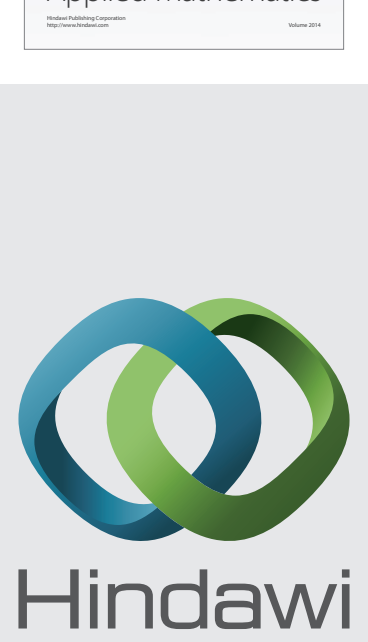

Submit your manuscripts at http://www.hindawi.com
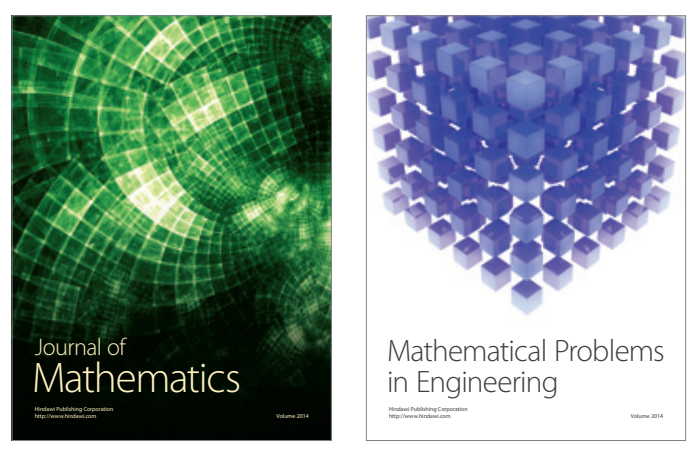

Mathematical Problems in Engineering
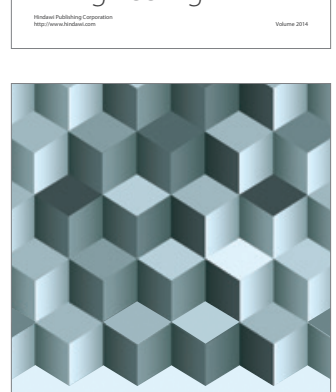

Journal of

Function Spaces
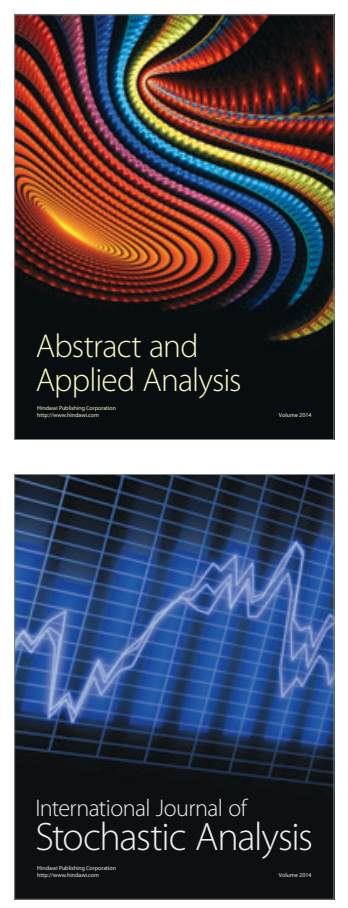

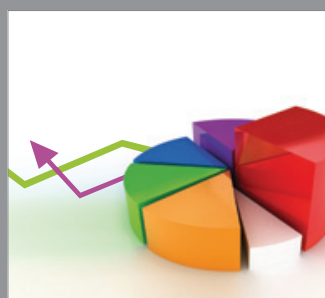

ournal of

Probability and Statistics

Promensencen
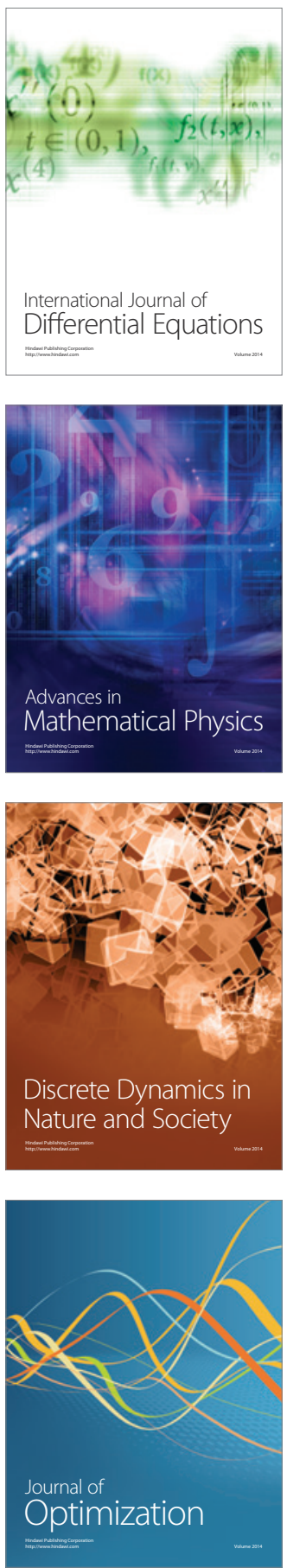